\title{
Multiple Organ Dysfunction Reduces In-Hospital Survival in COVID-19 Patients.
}

\author{
Najiba Abdulrazzaq, Kashif Bin Naeem *, Abdalla Alhajiri, Ayman Chkhis, Vinod Choondal, Mona Osman, \\ Kusay Almusa
}

Department of Internal Medicine and Cardiology, Al Kuwait Hospital, Dubai, UAE

Corresponding author: Kashif Bin Naeem; kbncardiology@yahoo.co.uk

Received 22 August 2020;

Accepted 04 September 2020;

Published 14 September 2020

\begin{abstract}
Background: Although primarily a respiratory illness, COVID-19 involves multiple organs when the disease is severe or critical. Hence, we conducted this study to evaluate the incidence of multiple organ dysfunction in COVID-19 patients and its implications on survival.

Methods: A retrospective analysis of laboratory-confirmed COVID-19 patients presenting to our center in Dubai, UAE between

April 2020 and July 2020. Data was collected from the electronic medical records and analyzed to evaluate multiple organ damage observed during hospital admission.

Findings: Five-hundred patients were studied. Overall mean age was 49.5 years (range 13-94), $76 \%$ males, $33 \%$ diabetics, $31 \%$ hypertensives. 97/500 (19.4\%) had evidence of single organ damage; $37 / 500$ (7.4\%) had two organ damage; and 105/500 (21\%) had more than two organ damage. Acute respiratory distress syndrome was the most prevalent organ damage, $153 / 500$ (30.6\%); followed by acute cardiac injury, $120 / 500$ (24\%); acute kidney injury $107 / 500$ (21.4\%); acute liver injury $96 / 500(19.2 \%)$; septic shock $93 / 500$ (18.6\%); disseminated intravascular coagulation $27 / 500(5.4 \%)$, and heart failure $17 / 500(3.4 \%)$. We found that in-hospital survival reduced as the number of organs involved increased; only $20 \%$ patients survived who had more than 2 organ damage. Also, the chances of survival reduced considerably once other organs were involved in addition to the acute respiratory distress syndrome (91.6\% survival in ARDS alone vs. 28.6\% survival in ARDS with acute kidney injury vs. $10.4 \%$ survival in ARDS with shock/acute cardiac injury/acute kidney injury).

Conclusion: Multiple organ dysfunction is common in COVID-19 as 21\% had evidence of more than two organ damage in our study. The survival in COVID-19 reduces significantly once multiple organs are involved. Early monitoring and recognition of multiple organ dysfunction is necessary to prevent adverse outcomes and improve survival.
\end{abstract}

Keywords: COVID-19, multiple organ dysfunction, in-hospital survival.

\section{Introduction}

Coronavirus disease 2019 (COVID-19) pandemic, caused by the Severe Acute Respiratory Syndrome Coronavirus 2 (SARS-Cov-2), has caused widespread morbidity and mortality across the globe since it was first reported in Wuhan, China in December $2019^{[1]}$. As of 13 Aug 2020, the total number of confirmed cases worldwide are 20,634,064 with 749,576 confirmed deaths, whereas, United Arab Emirates (UAE) has 63,212 confirmed cases with 358 deaths (Johns Hopkins Coronavirus Resource Center) ${ }^{[2]}$. The disease has demonstrated a wide spectrum of clinical patterns including asymptomatic forms to severe pneumonia, respiratory failure, multiorgan dysfunction, circulatory failure and death ${ }^{[3,4]}$. Potential mechanisms of injury include a hyperinflammatory response of the body together with the direct effects of severe acute respiratory syndrome on organs via angiotensin-converting enzyme 2 (ACE2) [5].
Understanding the potential organ injuries throughout the course of illness is crucial to guide early aggressive clinical management of these patients. Hence, we conducted this study where we explored the incidence of multiple organ dysfunction in COVID-19 patients and evaluated its impact on in-hospital survival.

\section{Methods}

The study was conducted at Al Kuwait Hospital, Dubai, UAE between April 2020 and July 2020. This involved a retrospective analysis of all consecutive patients admitted to our hospital with laboratory-confirmed COVID-19 disease. The laboratory used Sacace Real Time Reverse Transcription Polymerase Chain Reaction (rRT-PCR) test to diagnose COVID-19 disease. The test was performed on patients' nasopharyngeal swabs. RNA was extracted using SaMag Viral Nucleic Acid Extraction system. 
Extracted RNA was amplified using BGI- Real Time Fluorescent RT-PCR kit for the detection of COVID-19.

Data was extracted from the hospital electronic medical records retrospectively. This included basic demographics (age, sex), clinical data (symptoms, comorbidities), admission laboratory parameters (complete blood count, coagulation tests, inflammatory markers, renal function tests, cardiac and liver enzymes), admission chest radiography and chest computed tomography scan, multiple organ dysfunction, mechanical ventilation and death. All data was independently reviewed and entered into the computer excel sheet by two analysts.

Data is presented as counts and percentages for categorical variables. Groups were compared using statistical tests (chi-square for categorical variables and t-tests for comparing means). P-value $<0.05$ was considered statistically significant. Data was analyzed using Stat Plus statistical programme version 7 for Mac.

\section{Study definitions:}

Acute cardiac injury (ACI) was defined as the presence of highsensitivity troponin-I level above the 99th percentile upper reference limit, or if new abnormalities were shown in electrocardiography or echocardiography ${ }^{[3]}$. Acute respiratory distress syndrome (ARDS) was defined as per the Berlin Definition; the development of bilateral lung opacities (not fully explained by effusions, lobar/lung collapse, or nodules), respiratory failure not fully explained by cardiac failure or fluid overload and low $\mathrm{PaO} 2 / \mathrm{FiO} 2$, within 1 week of a known clinical insult or new or worsening respiratory symptoms ${ }^{[6]}$. Acute kidney injury (AKI) was defined as any of the following: increase in serum creatinine $\geq 0.3$ $\mathrm{mg} / \mathrm{dL}(\geq 26.5 \mathrm{umol} / \mathrm{L})$ within $48 \mathrm{hrs}$; or increase in serum creatinine $\geq 1.5$ times baseline, which is known or presumed to have occurred within the prior 7 days; or urine volume $<0.5$ $\mathrm{ml} / \mathrm{kg} / \mathrm{hr}$ for 6 hours ${ }^{[7]}$. Acute liver injury (ALI) was defined as the presence of high alanine amiotransferase (ALT) and/or high aspartate aminotransferase (AST) more than 5 times the upper normal limit. Septic shock was defined as a clinical construct of sepsis with persisting hypotension requiring vasopressors to maintain $\mathrm{MAP} \geq 65 \mathrm{~mm} \mathrm{Hg}$ and having a serum lactate level $>2$ $\mathrm{mmol} / \mathrm{L}(18 \mathrm{mg} / \mathrm{dL})$ despite adequate volume resuscitation ${ }^{[8]}$. Disseminated intravascular coagulation (DIC) was defined in the context of sepsis as an acquired syndrome characterized by activation of coagulation pathways, resulting in the formation of intravascular thrombi and depletion of platelets and coagulation factors, leading to decreased platelet count, increased prothrombin time and elevated d-dimers. Disease severity was classified as mild to moderate (non-pneumonia or mild pneumonia), severe (dyspnea, respiratory rate $\geq 30 / \mathrm{min}$, blood oxygen saturation $\leq 93 \%$, ratio of partial pressure of arterial oxygen $(\mathrm{PaO} 2)$ to fraction of inspired oxygen $(\mathrm{FiO} 2)<300$, lung infiltrates $>50 \%$ within $24-48$ hours), and critical (respiratory failure, septic shock, multiorgan dysfunction/failure) ${ }^{[9]}$.

\section{Results}

Five-hundred laboratory-confirmed COVID-19 patients were studied.

\section{Baseline characteristics:}

Table 1 describes the baseline clinical characteristics of 500 patients and reports the incidence of various organ damage with characteristics of each. Overall, mean age was 48.5 years with a range of 13-94 years. $76 \%$ were males; $33 \%$ were diabetics, $31 \%$ were hypertensives and $5 \%$ had history of cardiovascular disease. Admission chest radiography showed presence of bilateral airspace opacities in $64 \%$ of patients, while $78 \%$ showed bilateral ground glass or consolidative opacities in computed tomography (CT) chest. Laboratory tests on admission showed lymphopenia in 35\%, high D-dimers in 61\%, high ferritin in 57\% and high C-reactive protein in $85 \%$.

\section{Incidence of multiple organ dysfunction:}

Although acute respiratory distress syndrome was the most frequently organ dysfunction noted $(153 / 500,30.6 \%), 120 / 500$ (24\%) had acute cardiac injury, 107/500 (21.4\%) had acute kidney injury, and 96/500 (19.2\%) had acute liver injury. Septic shock was seen in $93 / 500$ (18.6\%), disseminated intravascular coagulation in $27 / 500(5.4 \%)$, and heart failure in $17 / 500$ (3.4\%). 96/500 (19.2\%) had one organ dysfunction, $37 / 500(7.4 \%)$ had 2 organs involved and 105/500 (21\%) had more than two organ dysfunction.

\section{Survival according to the number of organs damaged:}

We analyzed survival according to the number of organs involved. Table 2 shows the comparison of survivors versus non-survivors classified according to the number of organs affected. 98\% survived with one organ dysfunction, $64.8 \%$ survived with two organ damage, whereas only $20 \%$ survived if more than 2 organs were affected (Figure 1).

\section{Survival according to the type of organs damaged:}

We further analyzed survival in patients with ARDS alone and then survival with the type of organ involved additionally (Table 3). We found that $91.6 \%$ survived with ARDS alone, whereas $28.6 \%$ only survived if acute kidney injury was present with ARDS. Survival was reduced to $33.4 \%$ in the presence of shock. Only $10.4 \%$ survived in the presence of ARDS with shock, acute cardiac and kidney injury (Figure 2).

Table 1: Multiple organ dysfunction in 500 COVID-19 patients: clinical characteristics and outcomes.

\begin{tabular}{|c|c|c|c|c|c|c|c|c|}
\hline & $\begin{array}{l}\text { Total } \\
\text { sample size }\end{array}$ & ARDS & $\begin{array}{l}\text { Acute } \\
\text { cardiac } \\
\text { injury }\end{array}$ & $\begin{array}{l}\text { Heart } \\
\text { failure }\end{array}$ & $\begin{array}{l}\text { Acute } \\
\text { kidney } \\
\text { injury }\end{array}$ & $\begin{array}{l}\text { Acute } \\
\text { liver } \\
\text { injury }\end{array}$ & DIC & Shock \\
\hline No. /Total No. $(\%)$ & 500 & $\begin{array}{c}\text { 153/500 } \\
(30.6)\end{array}$ & $120 / 500(24)$ & $17 / 500(3.4)$ & $\begin{array}{c}107 / 500 \\
(21.4)\end{array}$ & $\begin{array}{c}96 / 500 \\
(19.2)\end{array}$ & $27 / 500(5.4)$ & 93/500 (18.6) \\
\hline \multicolumn{9}{|l|}{ Demographics } \\
\hline Age, mean (range) & $48.5(13-94)$ & $54.1(24-94)$ & $57.3(20-94)$ & $62(28-94)$ & $58.1(22-94)$ & $49.7(20-94)$ & $50.8(29-89)$ & $57.6(32-94)$ \\
\hline Male sex, no./total no. $(\%)$ & $380 / 500(76)$ & $137 / 153(90)$ & $102 / 120(85)$ & $14 / 17(82)$ & 96/107 (90) & $83 / 96(86)$ & $23 / 27(85)$ & $84 / 93(90)$ \\
\hline Diabetes, no./total no. (\%) & 165/500 (33) & $77 / 153(50)$ & $69 / 120(58)$ & 9/17 (53) & $61 / 107(57)$ & $34 / 96(35)$ & $8 / 27(30)$ & $55 / 93(59)$ \\
\hline
\end{tabular}




\begin{tabular}{|c|c|c|c|c|c|c|c|c|}
\hline $\begin{array}{l}\text { Hypertension, no./total no. } \\
(\%)\end{array}$ & $156 / 500(31)$ & $69 / 153(45)$ & $64 / 120(53)$ & 10/17 (59) & 61/107 (57) & $31 / 96(32)$ & 8/27 (30) & $49 / 93(53)$ \\
\hline CVD, no./total no. $(\%)$ & $27(5)$ & $14 / 153(9)$ & $16 / 120(13)$ & $3 / 17(18)$ & $11 / 107(10)$ & $6 / 96(6)$ & $0(0)$ & 9/93 (10) \\
\hline \multicolumn{9}{|l|}{ Imaging } \\
\hline $\begin{array}{l}\text { Bilateral opacities on CXR, } \\
\text { no./total no. }(\%)^{*}\end{array}$ & $322 / 500(64)$ & $135 / 153(88)$ & $95 / 120(79)$ & $12 / 17(70)$ & $85 / 107(79)$ & $81 / 96(84)$ & $22 / 27(82)$ & $79 / 93(85)$ \\
\hline $\begin{array}{l}\text { Bilateral opacities on CT } \\
\text { chest, no./total no. }(\%)^{*}\end{array}$ & $333 / 425(78)$ & $87 / 100(87)$ & $67 / 81(83)$ & $9 / 10(90)$ & $59 / 92(64)$ & $67 / 76(88)$ & $21 / 27(78)$ & $45 / 56(80)$ \\
\hline \multicolumn{9}{|l|}{ Admission laboratory results } \\
\hline $\begin{array}{l}\text { Lymphocytes }<1 \times 10^{3} / \mathrm{mcL} \text {, } \\
\text { no./total no. }(\%)^{*}\end{array}$ & $176 / 500(35)$ & $94 / 153(61)$ & $67 / 120(56)$ & $5 / 17(29)$ & $61 / 107(57)$ & $51 / 96(53)$ & $17 / 27(63)$ & $61 / 93(66)$ \\
\hline $\begin{array}{l}\text { Neutrophilia }>14 \times 10^{3} / \mathrm{mcL} \text {, } \\
\text { no./total no. }(\%)^{*}\end{array}$ & $42 / 500(8)$ & $24 / 153(16)$ & 23/120 (19) & 2/17 (12) & $22 / 107(21)$ & $10 / 96(10)$ & $1 / 27(4)$ & 18/93 (19) \\
\hline $\begin{array}{l}\text { Low platelets }<150 \\
\times 10^{3} / \mathrm{mcL} \text {, no./total no. }(\%)^{*}\end{array}$ & $53 / 500(11)$ & $23 / 153(15)$ & $13 / 120(11)$ & $3 / 17(18)$ & $14 / 107(13)$ & $13 / 96(14)$ & $27 / 27(100)$ & $13 / 93(14)$ \\
\hline $\begin{array}{l}\text { High PT }>12 \text { seconds, } \\
\text { no./total no. }(\%){ }^{*}\end{array}$ & $248 / 500(49)$ & $108 / 153(71)$ & $79 / 120(66)$ & $11 / 17(65)$ & 68/107 (64) & $61 / 96(64)$ & $27 / 27(100)$ & $68 / 93(73)$ \\
\hline $\begin{array}{l}\mathrm{eGFR}<60 \mathrm{ml} / \mathrm{min} / 1.73 \mathrm{~m}^{2} \text {, } \\
\text { no./total no. }(\%)\end{array}$ & $71 / 500(14)$ & $57 / 153(37)$ & $48 / 120(40)$ & $7 / 17(41)$ & 63/107 (59) & $21 / 96(22)$ & $9 / 27(33)$ & 43/93 (46) \\
\hline $\begin{array}{l}\text { ALT }>63 \text { IU/L / AST >37 } \\
\text { IU/L, no./total no. }(\%) *\end{array}$ & $271 / 500(54)$ & $103 / 153(67)$ & $76 / 120(63)$ & $10 / 17(59)$ & $70 / 107(65)$ & 76/96 (79) & 19/27 (70) & $65 / 93(70)$ \\
\hline $\begin{array}{l}\text { HS-Troponin I >60 ng/L, } \\
\text { no./total no. }(\%)^{*}\end{array}$ & 49/500 (10) & $33 / 153(22)$ & $51 / 120(43)$ & $10.17(59)$ & 26/107 (24) & $14 / 96(15)$ & $5 / 27(18)$ & $23 / 93$ \\
\hline $\begin{array}{l}\text { Pro-BNP >125 ng/L, } \\
\text { no./total no. }(\%)\end{array}$ & $228 / 358(64)$ & $121 / 140(86)$ & $83 / 92(90)$ & $14 / 14(100)$ & 83/91 (91) & $54 / 83(65)$ & $22 / 24(92)$ & $80 / 85(94)$ \\
\hline $\begin{array}{l}\text { D-dimer }>0.5 \mathrm{mg} / \mathrm{dL} \text {, } \\
\text { no./total no. }(\%)^{*}\end{array}$ & $304 / 500(61)$ & $132 / 153(86)$ & $105 / 120(88)$ & $17 / 17(100)$ & 95/107 (89) & $78 / 96(81)$ & $27 / 27(100)$ & $85 / 93(91)$ \\
\hline $\begin{array}{l}\text { Ferritin }>388 \mathrm{mcg} / \mathrm{L} \text {, } \\
\text { no./total no. }(\%)^{*}\end{array}$ & $285 / 500(57)$ & $127 / 153(83)$ & $93 / 120(78)$ & $13 / 17(76)$ & 86/107 (83) & $85 / 96(88)$ & $20 / 27(74)$ & 78/93 (84) \\
\hline $\begin{array}{l}\mathrm{CRP}>3 \mathrm{mg} / \mathrm{L}, \text { no./total no. } \\
(\%)^{*}\end{array}$ & $426 / 500(85)$ & $150 / 153(98)$ & $116 / 120(97)$ & $17 / 17(100)$ & $105 / 107(93)$ & 93/96 (97) & $26 / 27(96)$ & 90/93 (97) \\
\hline $\begin{array}{l}\text { Procalcitonin }>0.1 \mathrm{mcg} / \mathrm{L} \text {, } \\
\text { no./total no. }(\%)^{*}\end{array}$ & $201 / 500(40)$ & 109/153 (71) & $83 / 120(69)$ & 10/17 (59) & 77/107 (72) & $53 / 96(55)$ & $14 / 27(52)$ & $67 / 93(72)$ \\
\hline \multicolumn{9}{|l|}{ Outcomes } \\
\hline $\begin{array}{l}\text { Critical illness, no./total no. } \\
(\%)\end{array}$ & $145 / 500(29)$ & $\begin{array}{c}153 / 153 \\
(100)\end{array}$ & $99 / 120(83)$ & $12 / 17(671)$ & 88/107 (82) & $55 / 96(57)$ & $15 / 27(56)$ & 92/93 (99) \\
\hline $\begin{array}{l}\text { Mechanical ventilation, } \\
\text { no./total no. }(\%)\end{array}$ & $102 / 500(20)$ & $100 / 153(65)$ & $84 / 120(70)$ & $8 / 17(47)$ & 77/107 (72) & $43 / 96(45)$ & $9 / 27(33)$ & $85 / 93(91)$ \\
\hline Death, no./total no. (\%) & $99 / 500(20)$ & $98 / 153(64)$ & $82 / 120(68)$ & 9/17 (53) & 76/107 (71) & $40 / 96(42)$ & $9 / 27$ (33) & $82 / 93$ (88) \\
\hline
\end{tabular}

Data presented as counts and percentages.

ARDS, Acute Respiratory Distress Syndrome; DIC, Disseminated Intravascular Coagulation; CVD, Cardiovascular Disease; CXR, chest radiography; GGO, Ground Glass Opacifications; CT, computed tomography; PT, Prothrombin Time; eGFR, estimated-glomerular filtration rate; ALT, Alaninine Transaminase; AST, Aspartate Transaminase; HS-Troponin-I, High-Sensitivity-Troponin-I; Pro-BNP, Pro-B-type Natriuretic Peptide; CRP, C-Reactive Protein.

*Denotes investigations done upon admission. Upper limits of laboratory values are taken from local laboratory policies. 
Table 2: Comparison of survivors versus non-survivors classified according to the number of organs affected.

\begin{tabular}{|l|l|l|l|l|}
\hline Number of organs damaged & No. of patients & Survivors & Non-survivors & ${ }^{1}$ p-value for survivors vs. non-survivors \\
\hline Single organ * no./total no. (\%) & $97 / 500(19.4)$ & $95 / 97(98)$ & $2 / 97(2.1)$ & $<0.001$ \\
\hline Two organs *, no./total no. (\%) & $37 / 500(7.4)$ & $24 / 37(64.8)$ & $13 / 37(35)$ & 0.014 \\
\hline More than 2 organs *, no./total no. (\%) & $105 / 500(21)$ & $21 / 105(20)$ & $84 / 105(80)$ & $<0.001$ \\
\hline
\end{tabular}

Data presented as counts and percentages.

*Organs involved: lungs, heart, kidneys, liver, circulatory system, coagulation system.

1 p-value significant $<0.05$.

Table 3: Comparison of survivors versus non-survivors classified according to the type of organ affected.

\begin{tabular}{|l|l|l|l|l|}
\hline Organ damage & No. of patients & Survivors & Non-survivors & $\begin{array}{c}\text { 1 p-value for survivors } \\
\text { vs. non-survivors }\end{array}$ \\
\hline ARDS only, no./total no. (\%) & $24 / 500(4.8)$ & $22 / 24(91.6)$ & $2 / 24(8.4)$ & $<0.001$ \\
\hline ARDS + ACI, no./total no. (\%) & $9 / 500(1.8)$ & $4 / 9(44.5)$ & $5 / 9(55.5)$ & 0.007 \\
\hline ARDS + AKI, no./total no. (\%) & $7 / 500(1.4)$ & $2 / 7(28.6)$ & $5 / 7(71.4)$ & $<0.001$ \\
\hline ARDS + Shock, no./total no. (\%) & $3 / 500((0.6)$ & $1 / 3(33.4)$ & $2 / 3(66.6)$ & 0.04 \\
\hline ARDS + Shock + ACI, no./total no. (\%) & $8 / 500(1.6)$ & $2 / 8(25)$ & $6 / 8(75)$ & $<0.001$ \\
\hline ARDS + Shock + ACI + AKI, no./total no. (\%) & $29 / 500(5.8)$ & $3 / 29(10.4)$ & $26 / 29(89.6)$ & $<0.001$ \\
\hline ARDS + Shock + ACI + AKI + ALI, no./total no. (\%) & $26 / 500(5.2)$ & $1 / 26(3.9)$ & $25 / 26(96.1)$ & $<0.001$ \\
\hline
\end{tabular}

Data presented as counts and percentages.

ARDS, acute respiratory distress syndrome; ACI, acute cardiac injury; AKI, acute kidney injury; ALI, acute liver injury. 1 p-value significant $<0.05$.

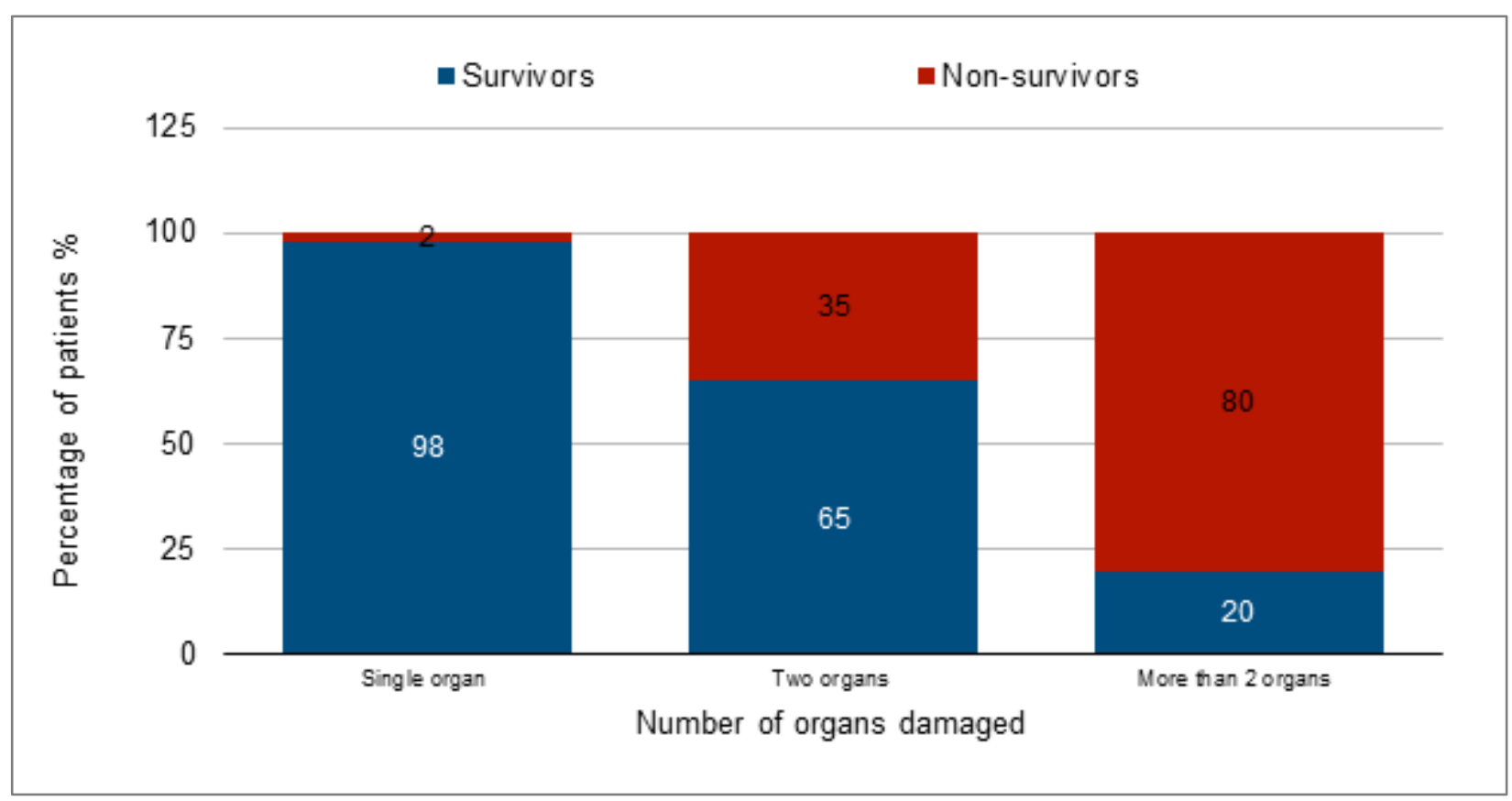

Figure 1: Bar chart comparing the proportion of survivors vs. non-survivors according to the number of organs involved. 


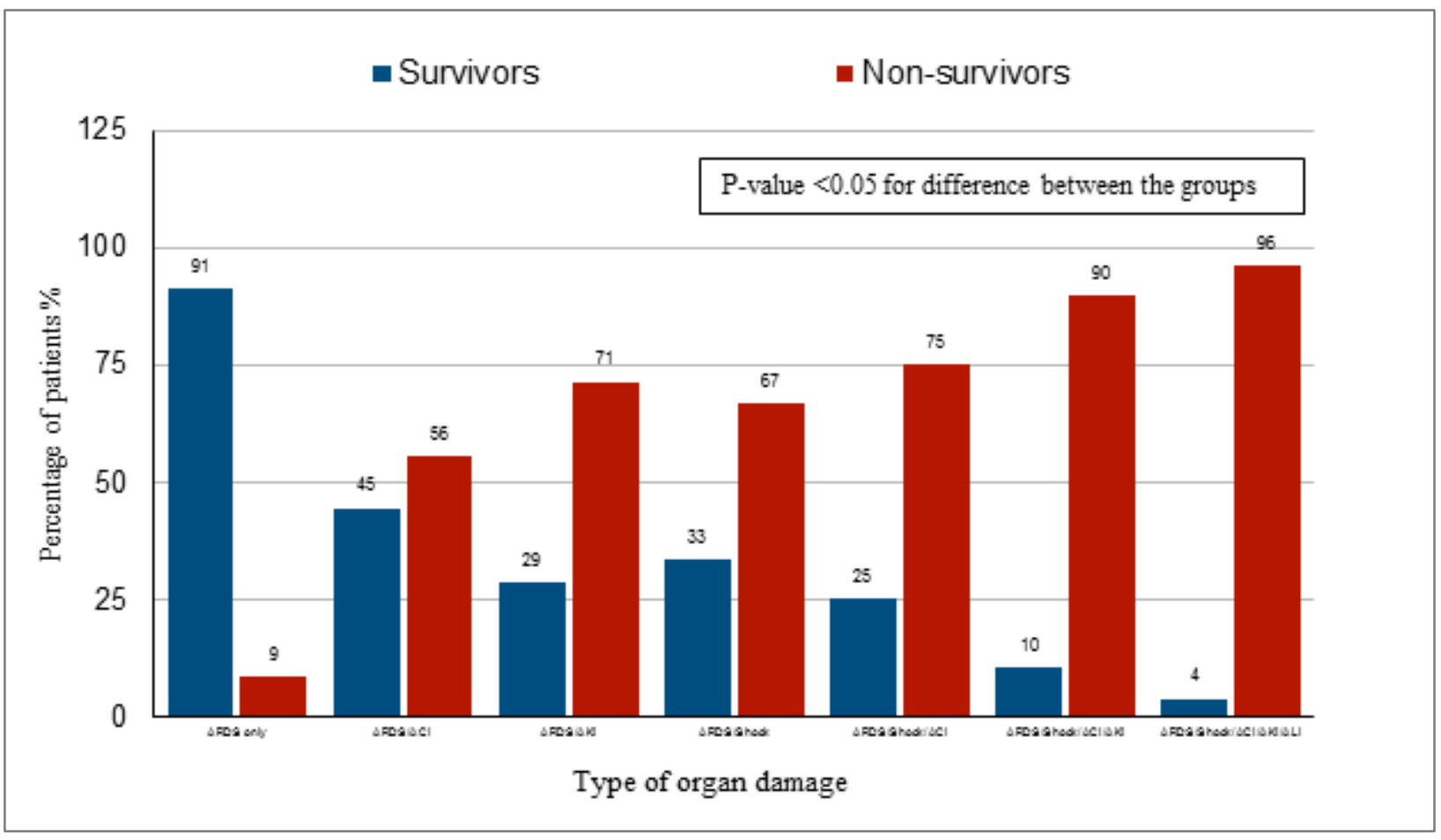

Figure 2: Bar chart comparing the proportion of survivors vs. non-survivors according to the type of organs involved.

\section{Discussion}

\section{Main findings of this study:}

This is a retrospective analysis of COVID-19 patients which evaluated the incidence of multiple organ involvement in the disease and further studied the effect on survival. Mean age of patients in our study was 48.5 years, highlighting a predominant middle-aged population. Multiple organ dysfunction was common. $7.4 \%$ had two-organ dysfunction, while $21 \%$ had more than twoorgan dysfunction. Mean age of patients who developed multiorgan dysfunction was less than 60 years, except heart failure where mean age was 62 years. Acute respiratory distress syndrome (ARDS) was the most common organ failure seen, found in $30.6 \%$ of our patients. This was expected in view of the primarily respiratory nature of disease. The most common organ involved after lungs was the heart, as $24 \%$ of our patients developed acute cardiac injury, that manifested with a rise in high-sensitivity troponin levels. This is alarming as only $13 \%$ of these patients had history of cardiovascular disease and may be assumed to have had a healthy cardiovascular system. Interestingly, $79 \%$ of these patients had abnormal chest radiograph on admission, hence revealing the high association between cardiac and lung injury in COVID-19. Acute kidney injury was found in $21.4 \%$ of our patients. Acute liver injury was found in $19.2 \%$ of our patients. Less frequent organ damage seen was heart failure $(3.4 \%)$ and disseminated intravascular coagulation (5.4\%). We also evaluated survival in patients with multiple organ dysfunction and found that the survival reduces significantly as more organs are affected. Figure 1 illustrates that the survival is reduced to only $20 \%$ once more than two organs are affected. Also, we found that patients with ARDS alone have favourable survival chance (91.6\%), but as other organs start failing on top of ARDS, the prognosis worsens. Figure 2 indicates how the presence of shock, acute kidney injury and acute cardiac injury in addition to ARDS reduces survival.

\section{What is already known on this topic:}

Although largely considered a respiratory illness, morbidity and mortality in COVID-19 increases considerably in the presence of multiple organ failure that involves vital organs like the heart, kidneys and liver. Possible causative mechanisms include the hyperinflammatory response of the body coupled with the direct effects of the severe acute respiratory syndrome on various organs via angiotensin-converting enzyme $2^{[5]}$. Increasing age, high Sequential Organ Failure Assessment (SOFA) score and D-dimer levels $>1 \mathrm{ug} / \mathrm{mL}$ on admission have been reported to be associated with mortality ${ }^{[10]}$. Although, the most frequent serious manifestation is pneumonia, Chen et al ${ }^{[11]}$ reported that $17 \%$ developed ARDS and among those, 65\% further deteriorated and died from multiple organ failure. Acute cardiac injury, manifested by either an increase in troponin levels or new electrocardiography or echocardiography abnormalities, has been reported in $7.2 \%$ by Wang et al ${ }^{[12]}$ with high case fatality rate as it is frequently associated with kidney injury, electrolyte disturbances and coagulation disorders. Although the exact mechanism of cardiovascular damage is not known, ACE2 dependent myocardial infection, cytokine storm from systemic inflammation and the hypoxic state from ARDS are proposed mechanisms of cardiac injury in COVID-19 ${ }^{[13]}$. Similarly, acute kidney injury during COVID-19 disease has been reported with an incidence ranging from $0.5 \%{ }^{[4]}$ to $19 \%{ }^{[14]}$. It is caused by acute tubular necrosis or direct renal cellular damage via ACE2 receptor related injury. Liver damage can also be seen in COVID-19, ranging from mild and transient injury to severe liver damage. Wong et al ${ }^{[15]}$ reported elevated levels of transaminases and bilirubin in 14.8-53.1\% of COVID-19 patients. Disseminated intravascular coagulation is another serious complication that is reported in $71.4 \%$ of nonsurvivors compared to only $0.6 \%$ of survivors ${ }^{[16]}$.

\section{What this study adds:}

This is rare data from the Middle East region. The study presents data on the incidence of multiple organ failure in COVID-19 patients and compares survivors versus nonsurvivors. Hence, this study will add significantly to the existing scientific knowledge that exists from other parts of the world.

\section{Limitations:}


This is a retrospective analysis of a relatively small sample size. Increasing number of patients with prospective analysis will provide additional information.

\section{Conclusion}

Although largely considered a respiratory illness, COVID-19 is associated with significant multiple organ dysfunction. The incidence is high, as $21 \%$ of patients in our study had more than two organ damage. The survival in COVID-19 reduces significantly once multiple organs are involved. Therefore, identifying and aggressively managing multiple organ dysfunction is necessary to prevent adverse outcomes and improve survival.

\section{Ethics approval and consent to participate}

The study was approved by the Ministry of Health and Prevention Research and Ethics Committee (approval reference no. MOHAP/DXB-REC/AAA/No. 39/2020), and informed consent was waived off.

\section{List of abbreviations}

COVID-19, Coronavirus disease 2019

SARS-Cov-2, Severe Acute Respiratory Syndrome Coronavirus 2

ACE2, angiotensin-converting enzyme 2

ARDS, Acute respiratory distress syndrome

\section{Data Availability}

The data that support the findings of the study are available from the corresponding author upon reasonable request.

\section{Conflict of interest}

The authors have no conflicts of interest to declare.

\section{Funding}

The study is not funded.

\section{Authors' contributions}

Design of the study: NA, KN, AA, VC.

Data collection: KN, AC, VC, MO, KA.

Analysis of data: NA, KN, VA, KA.

Writing of initial draft: NA, KN, AA.

Final revision of draft: NA, KN, AA, AC, KA.

All authors read and approved the final manuscript.

\section{References}

[1] Zhu N, Zhang D, Wang W, etal. China Novel Coronavirus Investigating and Research Team. A Novel Coronavirus from Patients with Pneumonia in China, 2019. N Engl J Med 2020;382:727-33.

[2] Johns Hopkins Coronavirus Resource Center. Center for Systems Science and Engineering (CSSE) at Johns Hopkins University, USA.

[3] Huang C, Wang Y, Li X, et al. Clinical features of patients infected with 2019 novel coronavirus in Wuhan, China. Lancet 2020 Feb 15; 395(10223): 497-506.

[4] Guan WJ, Ni ZY, Hu Y, et al. Clinical characteristics of coronavirus disease 2019 in China. N Engl J Med 2020.

[5] Zaim S, Chong JH, Sankaranarayanan V, Harky A. COVID-19 and Multiorgan Response. Curr Probl Cardiol 2020;45:100618.

[6] Acute Respiratory Distress Syndrome: The Berlin Definition, JAMA. 2012;307(23):2526-2533.

[7] Kidney Disease Improving Global Outcomes (KDIGO) Clinical Practice Guideline for Acute Kidney Injury. Kidney Int Suppl. 2012;2:1-138.

[8] The Third International Consensus Definitions for Sepsis and Septic Shock, JAMA, 2016;315(8):801-810.

[9] Novel Coronavirus Pneumonia Emergency Response Epidemiology Team. Vital surveillances: the epidemiological characteristics of an outbreak of 2019 novel coronavirus disease - China, 2020. China CDC Weekly.

[10] Weiss P, Murdoch DR. Clinical course and mortality risk of severe COVID-19. Lancet, 395 (2020), pp. 1014-1015.

[11] Chen J, Qi T, Liu L, et al. Clinical progression of patients with COVID-19 in Shanghai, China. J Infect (2020).

[12] Wang D, Hu B, Hu C, et al. Clinical characteristics of 138 hospitalized patients with 2019 novel coronavirusinfected pneumonia in Wuhan, China [published online ahead of print, $2020 \mathrm{Feb} 7]$. JAMA 2020:e201585.

[13] Aggarwal G, Cheruiyot I, Aggarwal S, et al. Association of Cardiovascular Disease with Coronavirus Disease 2019 (COVID-19) Severity: A Meta-Analysis. Curr Prob Cardiology 2020:100617.

[14] Arentz M, Yim E, Klaff L. Characteristics and outcomes of 21 critically ill patients with COVID-19 in Washington State. JAMA 2020.

[15] Wong SH, Lui RN, Sung JJ. Covid-19 and the digestive system. J Gastroenterol Hepatol. 2020.

[16] Tang N, Li D, Wang X, Sun Z. Abnormal coagulation parameters are associated with poor prognosis in patients with novel coronavirus pneumonia. J Thromb Haemost 2020;18:844-847. 